\title{
Average Energy Efficiency Contours for Single Carrier AWGN MAC
}

\author{
Amir Akbari, Muhammad Ali Imran, Reza Hoshyar, Rahim Tafazolli \\ Centre for Communication Systems Research, University of Surrey, UK \\ Email: \{a.akbari, m.imran, r.hoshyar, r.tafazolli\}@surrey.ac.uk
}

\begin{abstract}
Energy efficiency has become increasingly important in wireless communications, with significant environmental and financial benefits. This paper studies the achievable capacity region of a single carrier uplink channel consisting of two transmitters and a single receiver, and uses average energy efficiency contours to find the optimal rate pair based on four different targets: Maximum energy efficiency, a trade-off between maximum energy efficiency and rate fairness, achieving energy efficiency target with maximum sum-rate and achieving energy efficiency target with fairness. In addition to the transmit power, circuit power is also accounted for, with the maximum transmit power constrained to a fixed value. Simulation results demonstrate the achievability of the optimal energy-efficient rate pair within the capacity region, and provide the trade-off for energy efficiency, fairness and maximum sum-rate.
\end{abstract}

Index Terms-Capacity region, Contour, Energy efficiency, Multiple Access Channel, Uplink.

\section{INTRODUCTION}

Energy efficiency is an important aspect of future mobile communications systems, with significant environmental and financial benefits. Over the past decade, global warming has become an increasingly important subject, with information and communication technologies (ICT) causing around $2 \%$ of the world-wide $\mathrm{CO}_{2}$ emissions [1]. With the increasing interest in multimedia applications and high data rate services, it is very important to make sure that this percentage does not grow.

Due to the limitations in radio resources such as bandwidth and power, the concept of adaptive resource allocation has been thoroughly investigated in literature [2][4]. Earlier research has mainly focused on improving the throughput [3], [5], with limited work covering energy efficiency. The fundamental results of energy-efficient point to point links can be traced back to [6], which are further extended in [7], where the capacity in bits-per-joule is given for a single link on flat fading and frequency selective channels. Similar approaches are taken in [8] and [9], where the authors investigate uplink energy-efficient communications in OFDMA systems by improving the utilization of mobile energy in flat fading and frequency selective fading channels respectively. In [10], Cui et al. provide detailed analysis on the best modulation strategy to minimize the total energy consumption for sending a given number of bits. Both transmit and circuit powers are considered and the results show a considerable amount of savings in the total energy consumption, by optimizing the transmission time and modulation parameters.

Another topic thoroughly investigated in literature is channel capacity, which for a single-user case was shown in [11] to be the maximum possible data rate for a given channel with arbitrary small error probability. For the multiuser scenario, the capacity is defined as the Shannon capacity region [12], which corresponds to the set of rate vectors which all users can simultaneously maintain on the same channel with arbitrary small error probability. In [13], the author presents a thorough analysis on the Shannon capacity region for the downlink channel in both additive white Gaussian noise (AWGN) and fading channels. On the same topic, [14] shows that the Gaussian multiple access channel (MAC) and broadcast channel (BC) are duals of each other, provided the bi-directional links have the same channel gains and noise statistics in both directions, and the sum of individual power constraints in the uplink equals the power constraint in the downlink. In simple terms, the capacity region of the $\mathrm{BC}$ channel can be found if the capacity region of the MAC is only available, and vice versa.

In this paper, we start by revisiting the known formulation for the capacity region of the MAC and its dual $\mathrm{BC}$ channel for a two-user scenario. We then use average energy efficiency contours to find a trade-off between energy efficiency, fairness and maximum sum-rate. The rest of the paper is organized as follows: Section II briefly describes the system model and channel assumptions. Section III describes the capacity region for energy efficiency with variable power constraint. Section IV provides an overview on the fundamental characteristic of an energy-efficient system, and investigated its relation with the overall consumed power. Section V provides simulation results and discusses the achievability of the average energy efficiency contours by targeting energy efficiency, fairness and maximum sum-rate. And finally, the paper is concluded in section VI.

\section{SYSTEM MODEL}

Consider a single carrier uplink channel consisting of two transmitters and a single receiver, with each transmitter having power constraint $p_{k}$ and channel power gain $g_{k}$ (with $k=1,2$ ). Both transmitters and the receiver are assumed to have a single antenna, with the received signal being corrupted by AWGN with power spectral density (PSD) 
$\mathrm{N}_{0} / 2$. To simplify the design, we arbitrarily assume $g_{1}>g_{2}$ and that user 2 is always decoded first. The sum power and bandwidth are denoted as $P$ and $B$ respectively.

\section{CAPACITY REGION FOR ENERGY EFFICIENCY}

This section revisits the known formulation in literature with regards to the AWGN capacity region for MAC channels, [11], [12], and extends it from an energy efficiency perspective. To simplify the exposition of concepts, we will consider a 2 -user scenario, but the general properties and performance measures hold for larger number of users.

An optimal multiple access strategy in the uplink channel is for all users to spread their signal across the entire bandwidth. However, rather than decoding every user and treating the interference from other users as noise, a successive interference cancellation (SIC) receiver can be used to achieve capacity [15]. It is important to acknowledge the importance of the decoding order at the base station (BS) receiver, which will define the rate pairs achieved. In this paper, it is assumed that user 2 is decoded first, treating signals from user 1 as interference. The receiver can then subtract user 2's signal from the aggregate received signal and decode user 1 's data.

The rate of user $k$ was shown in [11] to be

$$
R_{k} \leq B \log _{2}\left(1+\frac{p_{k} g_{k}}{N_{0} B}\right), \quad k=1,2
$$

However, it should be noted that the sum of rates for all users cannot exceed the capacity of a point-to-point AWGN channel with received power equal to the sum of received powers from all users [16]. This is also demonstrated in the formulation for the capacity region of a 2-user Gaussian MAC which was shown in [12] to be

$$
\begin{aligned}
C_{M A C}(\boldsymbol{P} ; \boldsymbol{g})=\{ & \left(R_{1}, R_{2}\right): R_{1}+R_{2} \\
& \left.\leq B \log _{2}\left(1+\frac{p_{1} g_{1}+p_{2} g_{2}}{N_{0} B}\right)\right\}
\end{aligned}
$$

where $\boldsymbol{g}=\left(g_{1}, g_{2}\right)$ and $\boldsymbol{P}=\left(p_{1}, p_{2}\right)$ represent the channel gains and power constraints respectively.

In a standard point-to-point transmission, the capacity of a channel acts as an upper-bound (i.e. reliable communication is possible at rates less than the capacity). With two users in the system, this is extended to a capacity region, which contains the set of rate pairs such that both users can simultaneously transmit at rates $R_{1}$ and $R_{2}$. The set of achievable rates includes $\left(R_{l}, 0\right)$ and $\left(0, R_{2}\right)$, which corresponds to two extreme scenarios where one user transmits at its maximum rate, and the other remains silent.

The two constraints shown in (1) and (2) provide the capacity region of MAC in the form of a pentagon, shown in Fig. 1. Using the SIC receiver described before, user 1 can achieve its single-user bound with user 2 getting a nonzero rate, defined as $R_{2}{ }^{*}$.

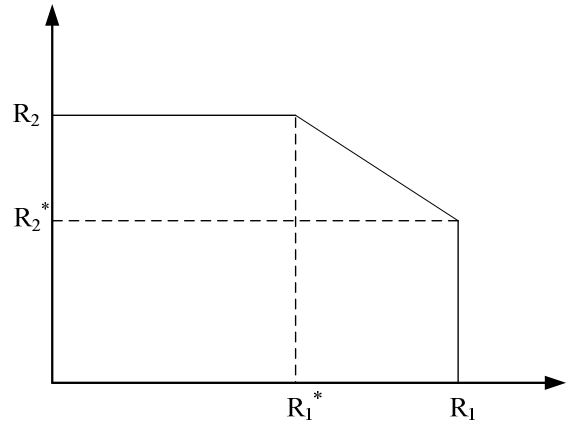

Figure 1. Capacity region of the two-user MAC AWGN channel

$$
\begin{aligned}
R_{2}^{*} & =B \log _{2}\left(1+\frac{p_{1} g_{1}+p_{2} g_{2}}{N_{0} B}\right)-B \log _{2}\left(1+\frac{p_{1} g_{1}}{N_{0} B}\right) \\
& =B \log _{2}\left(1+\frac{p_{2} g_{2}}{N_{0} B+p_{1} g_{1}}\right)
\end{aligned}
$$

By reversing the order of the cancellation, the same approach can be used to find $R_{I}{ }^{*}$.

$$
R_{1}^{*}=B \log _{2}\left(1+\frac{p_{1} g_{1}}{N_{0} B+p_{2} g_{2}}\right)
$$

As described before, rate pairs $\left(R_{1}, 0\right) /\left(0, R_{2}\right)$ represent the achievable rate vector when user one / two operates at its maximum rate, and user two/one is silent. Corner points $\left(R_{1}, R_{2}{ }^{*}\right)$ and $\left(R_{1}{ }^{*}, R_{2}\right)$ can also be found by employing a successive interference cancellation receiver. Without the constraint shown in (2), the capacity region would be a rectangle rather than a pentagon. It should be noted that any point along the line joining points $\left(R_{1}, R_{2}{ }^{*}\right)$ and $\left(R_{1}{ }^{*}, R_{2}\right)$ will maximize the sum rate.

For a fixed power constraint $\boldsymbol{P}$, the capacity region of the MAC is shaped as a pentagon. If we consider variable transmit power constraints (i.e. different power splits between users, with power allocation summing to the total power $P$ ) for the MAC users, then the capacity region will be formed from the union of all possible pentagons. This is also illustrated in Fig. 2, where the capacity region of the system with variable power constraints (shown in bold) is formed from the closed convex hull of the corner points of the capacity regions from the systems with fixed power constraints. Each pentagon in Fig.2 represents the MAC capacity region where $C_{M A C}\left(p_{1}, P-p_{1} ; g_{1}, g_{2}\right)$ is plotted for different values of $p_{I}$.

Instead of individual power constraints for users, we consider total power to be constrained for all uplink users and give them flexibility to adjust their maximum power limit (based on their channel/location) in order to improve the overall energy efficiency of the uplink system. This scenario is similar to the dual of BC channel, which was investigated in [14], and showed that the capacity region of a Gaussian BC with power constraint $P$ can be expressed as 
the union of the capacity regions of its dual MAC with power constraints $\left(p_{1}, p_{2}\right)$, where $p_{1}+p_{2}=P$.

$$
C_{B C}(P ; \boldsymbol{g})=\bigcup_{p_{1}+p_{2}=P} C_{M A C}\left(p_{1}, p_{2} ; \boldsymbol{g}\right)
$$

At the first glance, the AWGN uplink (MAC) and downlink (BC) channels appear to be very similar. However, there are three fundamental differences which make the capacity regions quite different. Firstly, multiple users are transmitting in the uplink, each with a separate power constraint, whereas in the downlink, a single power constraint is available. Secondly, there is one additive noise term in the uplink (since there is only one receiver), but in the downlink, each receiver has a noise term associated with it. And thirdly, the signal and noise associated with each user in the uplink travel through different channels, whereas in the downlink, both travel through the same channel [16].

The duality relationship shown in (5) holds, provided the bi-directional links have the same channel gain and noise statistics in both directions, and the sum of individual power constraints in the uplink equals the power constraint in the downlink.

\section{ENERGY EFFICIENCY}

This section gives an overview on the fundamental characteristic of an energy-efficient system, by investigating the bits-per-joule measure of a single carrier system and showing its relationship with the overall consumed power.

Following the assumption of using an SIC receiver and decoding user 2's data first, the transmit power of user 1 and 2 can be expressed as a function of their data transmission rates as

$$
\begin{gathered}
p_{1}=\frac{\left(2^{R_{1} / B}-1\right) N_{0} B}{g_{1}} \\
p_{2}=\frac{\left(2^{R_{2} / B}-1\right)\left(N_{0} B+p_{1} g_{1}\right)}{g_{2}}
\end{gathered}
$$

where the interference factor form user 1's signal affects user 2's transmit power.

For a channel with average power of $p_{k}$ watts and a channel capacity of $R_{k} \mathrm{bits} / \mathrm{sec}$, the energy efficiency is defined as $R_{k} / p_{k}$ bits/joule [7]. However, in addition to the transmit power, some power is consumed in the circuitry or dissipated in the form of heat. We shall account for this power as the circuit power, $P_{c}$, which is independent of the transmission state.

The overall energy efficiency of user $k$ is defined as

$$
E E_{k}=\frac{R_{k}}{P_{c}+p_{k}}, \quad k=1,2
$$

It should be noted that in an uplink scenario, multiple transmitters send data towards a single receiver, therefore each transmitter introduces an individual circuit power. It is assumed throughout this paper that all transmitters have equal circuit power.

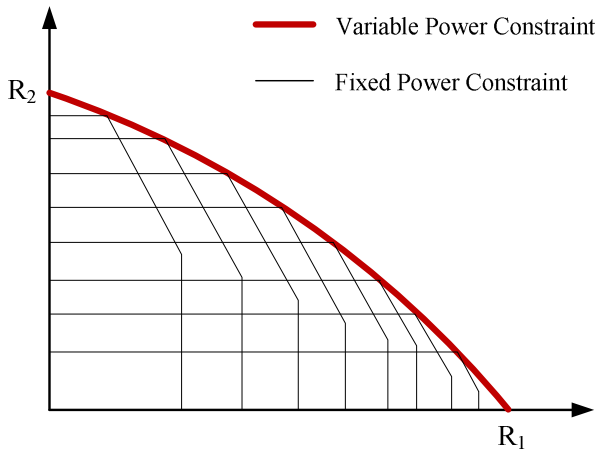

Figure 2. AWGN MAC capacity region of 2-user system with fixed and variable power constraints

Fig. 3 shows the relationship between overall consumed power (i.e. $P_{c}+P$ ) and energy efficiency for a single user, single carrier system. It is clearly seen that the energy efficiency increases as the overall consumed power is increased. However, this is true up to a certain power, after which the energy efficiency starts to decrease. This is the main idea behind energy-efficient systems, where, a continuous increase in the transmit power will decrease the energy efficiency. Therefore, maximum energy efficiency is achieved by tuning the power according to the rate requirements of the system. This paper focuses on a single carrier scenario, and can be extended to multicarrier systems as part of future work.

\section{SIMULATION RESUlTS AND DisCUSSIONS}

Section III provided the fundamental characteristics of the MAC and its dual BC channel, and specified the capacity region associated with each model. This was followed by section IV where, energy efficiency was defined as the bits-per-joule measure and the general relationship between consumed power and energy efficiency was graphically illustrated. This section presents

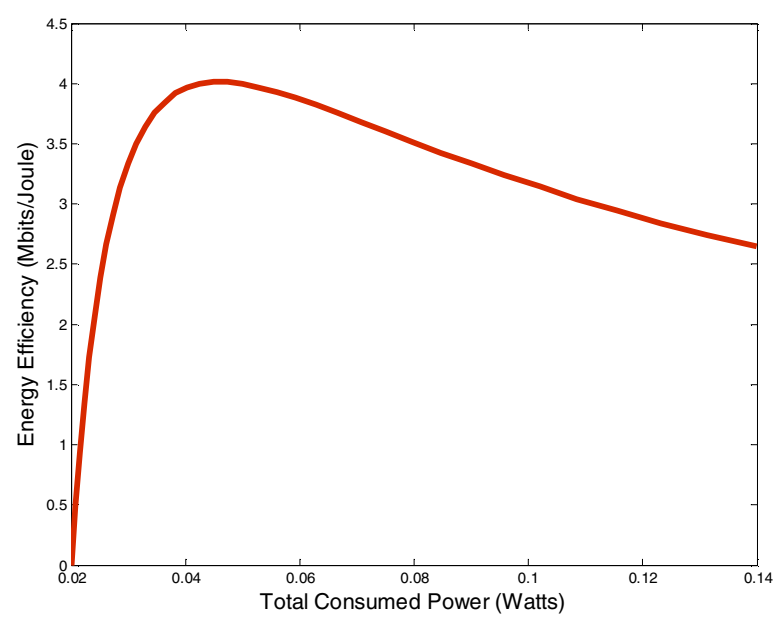

Figure 3. Relationship of consumed power and energy efficiency for a single user, single carrier scenario ( $\mathrm{B}=100 \mathrm{kHz}, \mathrm{g}=0.01, \mathrm{~N}_{0}=10^{-9} \mathrm{~W} / \mathrm{Hz}, P_{c}=0.02$ Watts $)$ 
the average energy efficiency contours for a single carrier AWGN MAC and identifies the optimal rate pair based on the system requirements. The system considered is a twouser AWGN MAC with a total transmit power of $20 \mathrm{~mW}$. The channel gains of user one and two are defined as $g_{l}=0.008$ and $g_{2}=0.005$ respectively. The system bandwidth is $B=100 \mathrm{KHz}$ and the receiver noise has $N_{0}=10^{-9} \mathrm{~W} / \mathrm{Hz}$.

As shown earlier, the capacity region of a Gaussian BC channel can be expressed as the union of the capacity regions of its dual MAC. This is also illustrated in Fig.2, where the closed convex hull of the corner points of the dual MAC form the BC capacity region. For the purpose of clarity, the uplink capacity regions (pentagons) will be omitted, and only the dual downlink boundary will be shown in the figures presented in this section.

The total transmit power is set to $P$, and split between user one and two as

$$
\boldsymbol{P}=[\alpha P,(1-\alpha) P]^{T}
$$

where $\alpha$ can take any real value within the interval $[0,1]$. Each power allocation gives a rate pair, and the union will form the rate region boundary.

If $E E_{1}$ and $E E_{2}$ denote the energy efficiency of user one and two respectively, then the average energy efficiency is defined as

$$
E E_{A V}=\frac{E E_{1}+E E_{2}}{2}
$$

The energy efficiency contours are obtained by plotting all rate pairs matching the requirement shown in (9), where each $E E_{A V}$ value will have its corresponding contour.

Fig. 4 presents the uplink capacity region (shown in bold dashed lines), energy efficiency contours and the optimal energy-efficient rate pair (converged to a point, marked with a cross).

Having identified the capacity region of interest and average energy efficiency contours, the next stage is to locate the optimal operating point using several criteria of optimality, which are discussed in subsequent sections.

\section{A. Maximum Energy Efficiency}

This approach reflects scenarios where the only target of the system is to maximize energy efficiency, without any concerns on fairness or sum-rate. The desired rate pair is chosen within the capacity region such that this target is met.

$$
\begin{aligned}
& \max _{R_{1}, R_{2}}\left(E E_{A V}\right) \\
& \text { s.t. }\left(R_{1}, R_{2}\right) \in C_{B C}(P ; \boldsymbol{g})
\end{aligned}
$$

Constraint (12) specifies that all rate pairs should be chosen within the capacity region boundary. Any segment of the energy efficiency contours that reach outside the capacity region will not be achievable.

For the example plotted in Fig.4, the maximum energyefficient point lies within the capacity region. Since the total transmit power defines the capacity region boundary, increasing or decreasing it will determine how far from the

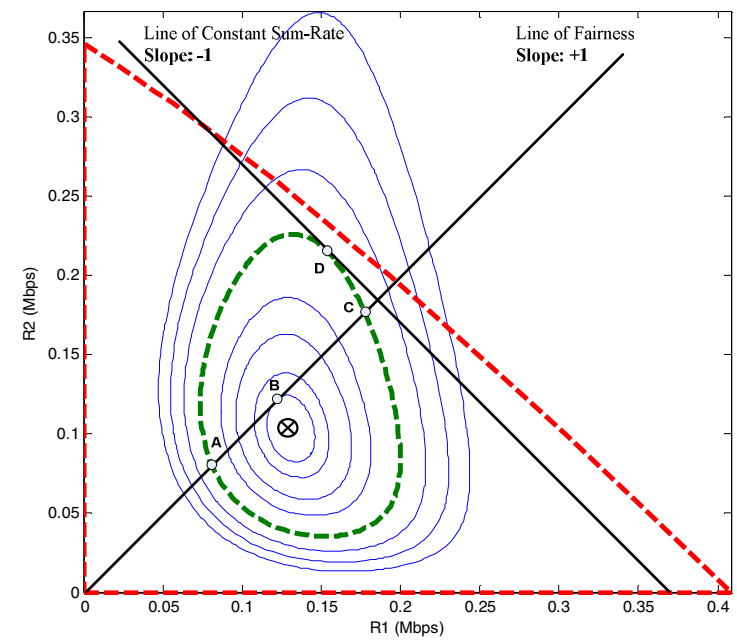

Figure 4. Energy efficiency, fairness and maximum sum-rate trade-off

capacity region boundary the energy-efficient point will be. In other words, by increasing the maximum transmit power, the capacity region will expand, and its boundary will be further away from the desired point. This is also true if we decrease the power, which will lead to a capacity region below the optimal point, which will not be achievable.

\section{B. Maximum Energy Efficiency and Rate Fairness}

It was shown in section $A$ that depending on the choice of parameters, the optimal energy-efficient rate pair can be found within the capacity region of the AWGN MAC. This section investigates scenarios where the points of interest are those that consider rate fairness between users as well as achieving the maximum possible energy efficiency.

$$
\begin{aligned}
& \max _{R_{1}, R_{2}}\left(E E_{A V}\right) \\
& \text { s.t. }\left(R_{1}, R_{2}\right) \in C_{B C}(P ; \boldsymbol{g}) \\
& \quad R_{1}=R_{2}
\end{aligned}
$$

Points with equal rate share for both users $\left(R_{I}=R_{2}\right)$ lie on a line passing through the origin with a slope of +1 , which we will refer to as the line of fairness. Based on the chosen system parameters, it can be seen in Fig.4 that the line of fairness does not pass through the optimal energy efficient point. Therefore, the maximum possible rate-fair, energyefficient value will be the point on the energy efficiency contour tangentially touching the line of fairness, shown as point $\mathbf{B}$ in Fig.4.

\section{Target Energy Efficiency with Maximum Sum-Rate}

Each of the contours shown in Fig.4 corresponds to an energy efficiency value. In other words, for a fixed energy efficiency target, only one of the contours will be of interest to the system, which we will refer to as the target energy efficiency $\left(E E_{T}\right)$, shown dashed in Fig.4. The main target of this section is to maximize the sum-rate by achieving a fixed energy efficiency target. 


$$
\begin{array}{ll}
\max \left(\sum_{\mathrm{i}=1}^{2} \mathrm{R}_{\mathrm{i}}\right) \\
\text { s.t. } & \left(R_{1}, R_{2}\right) \in C_{B C}(P ; \boldsymbol{g}) \\
& E E_{A V}=E E_{T}
\end{array}
$$

A line with the slope of -1 is shown in Fig.4 (line of constant sum-rate), where it contains all points which have the same sum-rate. The tangential point of this line and the capacity region boundary will give the maximum achievable sum-rate. In order to maximize sum-rate and be able to obtain $E E_{T}$ at the same time, we find the tangential point of the line of constant sum rate with the target contour. This point will present the rate pair achieving the target energy efficiency and having the maximum possible sum-rate, and is shown as point $\mathbf{D}$ in Fig.4. It should be noted that, another line with a slope of -1 can be drawn tangent to the target energy efficiency contour. Although this point meets the target energy efficiency value, it achieves a lower sum-rate compared to point $\mathbf{D}$.

\section{Target Energy Efficiency with Rate Fairness}

As seen in section $B$, in order to have rate fairness between users, the rate pair should be chosen along a line with a slope of -1 . For scenarios with a fixed energy efficiency target, all points along the contour corresponding to $E E_{T}$ will achieve the desired energy efficiency value. Assume the same $E E_{T}$ as section $C$, but with the intention of having rate fairness rather than maximum sum-rate.

$$
\begin{array}{ll}
\max \left(\sum_{\mathrm{i}=1}^{2} \mathrm{R}_{\mathrm{i}}\right) \\
\text { s.t. } & \left(R_{1}, R_{2}\right) \in C_{B C}(P ; \boldsymbol{g}) \\
& E E_{A V}=E E_{T} \\
& R_{1}=R_{2}
\end{array}
$$

The intersection of the line of fairness and the desired contour will give us the optimal operating point, which can be seen in Fig. 4 to be points $\mathbf{A}$ and $\mathbf{C}$. Both these points will meet the required criteria in terms of fairness and energy efficiency, however, the obvious choice in this case would be to operate at point $\mathbf{C}$, since it also has a higher maximum sum rate. Furthermore, both points $\mathbf{D}$ and $\mathbf{C}$ provide the same average energy efficiency, with $\mathbf{D}$ maximizing the sum rate and $\mathbf{C}$ ensuring fairness (i.e. $\mathrm{R}_{1}=\mathrm{R}_{2}$ ).

\section{CONCLUSION}

In this paper, we study the achievable capacity region of a single carrier AWGN MAC, consisting of two transmitters and a single receiver. We then take into account the duality criteria and show the capacity region of the Gaussian BC as the union of its dual MAC. Energy efficiency is defined as the bits-per-joule measure and the general relationship between overall consumed power and energy efficiency is given. We incorporate the two concepts and use average energy efficiency contours to find the optimal rate pair using several optimality criteria. Simulation results verify that an optimal energy-efficient rate pair can be found within the capacity region of the AWGN MAC. We show that points with equal rate share for both users lie on a line passing through the origin with a slope of +1 , which is referred to as the line of fairness. We also show that all point along a line with a slope of -1 have the same sum-rate. We demonstrate that with a fixed energy efficiency target, depending on the requirements of the system, both fairness and maximum sum-rate can be incorporated into the system. The work presented in this paper will be further extended to the multiuser $(K>2)$ scenario for future work, which will also investigate all possible decoding orders.

\section{ACKNOWLEDGMENT}

The research leading to these results has received partial funding from the European Community's Seventh Framework Programme [FP7/2007-2013] under grant agreement $n^{\circ} 247733$ - project EARTH.

The authors would also like to thank Dr. Fabien Heliot at CCSR, University of Surrey, for his insightful comments and helpful discussions.

\section{REFERENCES}

[1] S. Vadgama, "Trends in green wireless access," Fujitsu Scientific \& Technical Journal (FSTJ), vol. 45, no. 4, pp. 404-408, Oct 2009.

[2] C. Wong, R. Cheng, K. Lataief, R. Murch, " Multiuser OFDM with adaptive subcarrier, bit, and power allocation”, IEEE Journal on Selected Areas in Communications, vol. 17, no.10, pp. 1747-1758, Oct. 1999.

[3] W. Rhee, J. Cioffi, "Increase in capacity of multiuser OFDM system using dynamic subchannel allocation", in proc. IEEE Vehic. Tech. Conf., Tokyo, Japan, pp. 1085-1089, May 2000.

[4] N. Y. Ermolova, B. Makarevitch, "Low complexity adaptive power and subcarrier allocation for OFDMA", IEEE Trans. on Wireless Commun., vol. 6, no. 2, February 2007.

[5] T. Keller and L. Hanzo, "Adaptive multicarrier modulation: a convenient framework for time frequency processing in wireless communications, " in proc. of IEEE, vol. 88, pp. 611-640, May 2000

[6] M. J. E. Golay, "Note on the theoretical efficiency of information reception with PPM," in Proc. IRE 1949, vol. 37, no. 1, pp.1031, Sep. 1949.

[7] H. M. Kwon, T. G. Birdsall, "Channel Capacity in Bits Per Joule," IEEE Journal of Oceanic Engineering, vol. 0E-11, no. 1, pp.97-99, Jan. 1986.

[8] G. W. Miao, N. Himayat, Y. Li, D. Bormann, "Energy-efficient design in wireless OFDMA," in proc. IEEE ICC 2008, pp.33073312, May 2008.

[9] G. W. Miao, N. Himayat, Y. Li, "Energy-Efficient Transmission in Frequency-Selective Channels," in proc. IEEE GLOBECOM 2008, pp. 1-5, Nov. 2008.

[10] S. Cui, A. J. Goldsmith, A. Bahai, "Energy-constrained modulation optimization," IEEE Trans. Wireless Commun., vol. 4, no. 5, pp.2349-2360, Sep. 2005

[11] C. E. Shannon and W. Weaver, A Mathematical Theory of Communication. Urbana, IL: University Illinois Press, 1949.

[12] T. Cover and J. Thomas, Elements of Information Theory. New York: Wiley, 1991.

[13] A. J. Goldsmith, "The capacity of downlink fading channels with variable rate and power", IEEE Transactions on Vehicular Technology, vol. 46, no.3, pp. 1747-1758, Aug. 1997.

[14] N. Jindal, S. Vishwanath and A. J. Goldsmith, "On the duality of Gaussian multiple-access and broadcast channels", IEEE Transactions on information Theory, vol. 50, no.5, pp. 768-783, May 2004.

[15] D. Tse and P. Viswanath, Fundamentals of Wireless Communication. Cambridge University press, 2005.

[16] A. Goldsmith, Wireless Communications. Cambridge University press, 2005. 